\title{
Multi-band high resolution spectroscopy rules out the hot Jupiter BD+20 1790b
}

\section{First data from the GIARPS Commissioning}

I. Carleo ${ }^{1,2}$, S. Benatti ${ }^{2}$, A. F. Lanza ${ }^{3}$, R. Gratton ${ }^{2}$, R. Claudi ${ }^{2}$, S. Desidera ${ }^{2}$, G. N. Mace ${ }^{4}$, S. Messina $^{3}$, N. Sanna ${ }^{5}$, E. Sissa ${ }^{2}$, A. Ghedina 6 , F. Ghinassi ${ }^{6}$, J. Guerra ${ }^{6}$, A. Harutyunyan ${ }^{6}$, G. Micela ${ }^{7}$, E. Molinari ${ }^{6}, 16$, E. Oliva ${ }^{5}$, A. Tozzi $^{5}$, C. Baffa $^{5}$, A. Baruffolo ${ }^{2}$, A. Bignamini ${ }^{8}$, N. Buchschacher ${ }^{9}$, M. Cecconi ${ }^{6}$, R. Cosentino ${ }^{6}$, M. Endl, ${ }^{4}$, G. Falcini ${ }^{5}$, D. Fantinel ${ }^{2}$, L. Fini ${ }^{5}$, D. Fugazza ${ }^{10}$, A. Galli ${ }^{6}$, E. Giani ${ }^{5}$, C. González ${ }^{6}$, E. González-Álvarez ${ }^{7}, 11$, M. González $^{6}$, N. Hernandez ${ }^{6}$, M. Hernandez Diaz ${ }^{6}$, M. Iuzzolino ${ }^{5,12}$, K. F. Kaplan ${ }^{4}$, B. T. Kidder ${ }^{4}$, M. Lodi ${ }^{6}$, L. Malavolta ${ }^{1}$, J. Maldonado ${ }^{7}$, L. Origlia ${ }^{13}$, H. Perez Ventura ${ }^{6}$, A. Puglisi ${ }^{5}$, M. Rainer ${ }^{10}$, L. Riverol ${ }^{6}$, C. Riverol ${ }^{6}$, J. San Juan ${ }^{6}$, S. Scuderi ${ }^{3}$, U. Seemann ${ }^{14}$, K. R. Sokal ${ }^{4}$, A. Sozzetti ${ }^{15}$, and M. Sozzi ${ }^{5}$

\footnotetext{
${ }^{1}$ Dipartimento di Fisica e Astronomia, “Galilei”- Universtà degli Studi di Padova, Vicolo dell’Osservatorio 3, 35122 Padova, Italy e-mail: ilaria.carleo@oapd.inaf.it

2 INAF - Osservatorio Astronomico di Padova, Vicolo dell'Osservatorio 5, 35122 Padova, Italy

${ }^{3}$ INAF - Osservatorio Astrofisico di Catania, Via S. Sofia 78, 95123 Catania, Italy

${ }^{4}$ Department of Astronomy and McDonald Observatory - The University of Texas at Austin, 2515 Speedway, Stop C1400, Austin, TX 78712-1205, USA

${ }^{5}$ INAF - Osservatorio Astrofisico di Arcetri, Largo Enrico Fermi 5, 50125 Firenze, Italy

${ }^{6}$ Fundación Galileo Galilei - INAF, Rambla José Ana Fernandez Pérez 7, 38712 Breña Baja, TF, Spain

${ }^{7}$ INAF - Osservatorio Astronomico di Palermo, Piazza del Parlamento 1, 90134 Palermo, Italy

8 INAF - Osservatorio Astronomico di Trieste, via Tiepolo 11, 34143 Trieste, Italy

9 Département d'Astronomie - Université de Genève, Chemin des Maillettes, 511290 Versoix, Switzerland

${ }^{10}$ INAF - Osservatorio Astronomico di Brera, Via E. Bianchi 46, 23807 Merate (LC), Italy

${ }_{11}$ Dipartimento di Fisica e Chimica - Università degli Studi di Palermo,Via Archirafi 36, 90123 Palermo, Italy

12 Officina Stellare S.r.l., Via Della Tecnica, 87/89, 36030 Sarcedo (VI), Italy

${ }^{13}$ INAF - Osservatorio Astronomico di Bologna, Via Gobetti 93/3, 40129, Bologna, Italy

${ }^{14}$ Institut für Astrophysik - Georg-August-Universität Göttingen, Friedrich-Hund-Platz 1, 37077 Göttingen, Germany

15 INAF - Osservatorio Astrofisico di Torino, Via Osservatorio 20, 10025, Pino Torinese (TO), Italy

${ }^{16}$ INAF - Osservatorio Astronomico di Cagliari, Via della Scienza 5, 09047, Selargius (CA), Italy
}

Received 23 November 2017 / Accepted 16 April 2018

\section{ABSTRACT}

Context. Stellar activity is currently challenging the detection of young planets via the radial velocity (RV) technique.

Aims. We attempt to definitively discriminate the nature of the RV variations for the young active K5 star BD+20 1790, for which visible (VIS) RV measurements show divergent results on the existence of a substellar companion.

Methods. We compare VIS data with high precision RVs in the near-infrared (NIR) range by using the GIANO-B and IGRINS spectrographs. In addition, we present for the first time simultaneous VIS-NIR observations obtained with GIARPS (GIANO-B and HARPS-N) at Telescopio Nazionale Galileo (TNG). Orbital RVs are achromatic, so the RV amplitude does not change at different wavelengths, while stellar activity induces wavelength-dependent RV variations, which are significantly reduced in the NIR range with respect to the VIS.

Results. The NIR radial velocity measurements from GIANO-B and IGRINS show an average amplitude of about one quarter with respect to previously published VIS data, as expected when the RV jitter is due to stellar activity. Coeval multi-band photometry surprisingly shows larger amplitudes in the NIR range, explainable with a mixture of cool and hot spots in the same active region. Conclusions. In this work, the claimed massive planet around BD+20 1790 is ruled out by our data. We exploited the crucial role of multi-wavelength spectroscopy when observing young active stars: thanks to facilities like GIARPS that provide simultaneous observations, this method can reach its maximum potential.

Key words. instrumentation: spectrographs - techniques: radial velocities - planets and satellites: general - stars: activity stars: individual: $\mathrm{BD}+201790$

\section{Introduction}

The detection of giant planets around young stars can address key questions in the astrophysics of planetary formation and migration. In fact, looking at young stars provides an opportunity to observe the architecture of planets in their infancy. A variety of physical processes, such as planet-disc interaction, the Kozai mechanism (Kozai 1962), planet-planet 
scattering (Baruteau et al. 2014), or in-situ formation (Batygin et al. 2016), responsible for generating hot Jupiters (HJs), are expected to produce observable effects, including differences in orbital parameters (eccentricity and/or obliquity), migration timescales, an age-dependent frequency of such systems, and differences in the atmospheric composition of hot Jupiters across stellar ages. The investigation of the formation and migration histories may be carried out for example via intensive radial velocity (RV) monitoring of a sample of young stars, finding new planets and testing possible differences in the frequency of hot Jupiters with age. However, the high level of stellar activity characterizing young stars induces RV variations able to mimic planetary signals, demanding a specific processing in order to investigate its actual contribution. Recently, noteworthy results have been obtained through the modelling of stellar activity as correlated noise in a set of data with the Gaussian processes regression (see e.g. Haywood et al. 2014; Damasso et al. 2018). The first pioneering detection of a young $\mathrm{HJ}$ with the RV technique was recently announced around the weak-line T Tauri star V830 Tau using spectropolarimetry (Donati et al. 2016; Yu et al. 2017; Donati et al. 2017), after years of attempts marred by difficulties in dealing with the identification of planetary signals (both spectroscopic and photometric) in the presence of very high levels of activity of their young hosts. Simultaneous multi-band high resolution spectroscopy (HRS), for example in the visible (VIS) and near-infrared (NIR, 700-2500 nm) ranges, is a powerful tool for disentangling wavelength-dependent activity-induced RV signals (the impact of activity on RVs is expected to be typically three times lower in the NIR than in the VIS; see Prato et al. 2008; Mahmud et al. 2011; Crockett et al. 2012) from those Keplerian that are achromatic. An emblematic example of this principle is represented by the case of the HJ around TW Hya claimed by Setiawan et al. (2008), which was ruled out by NIR observations (Figueira et al. 2010a).

The building of new instrumentation allowing high precision HRS in the NIR range has been mainly pushed by the RV search of potentially habitable rocky planets around Mdwarf stars (see e.g. Reiners et al. 2010; Bonfils et al. 2013 and references therein). Some of those instruments, such as CARMENES (520-1710 nm, with spectral resolutions $R=$ 80 000-100 000, Quirrenbach et al. 2014; Reiners et al. 2018) or HARPS+NIRPS $(0.95-1.8 \mathrm{~nm}, R \sim 100000$, Conod et al. 2016), also guarantee contemporary VIS-NIR observations. In this framework, the GIARPS (GIANO-B + HARPS-N) project (Claudi et al. 2017) has been conceived precisely to exploit the full potential of the simultaneous VIS and NIR HRS technique, allowing us to have the two high resolution spectrographs of the Telescopio Nazionale Galileo (TNG, La Palma) working simultaneously: HARPS-N (High Accuracy Radial velocity Planet Searcher for the Northern hemisphere) in the visible and GIANO-B in the NIR (see details in Sect. 2). The RV precisions achievable by the VIS and NIR channels of GIARPS (see more details in Sect. 2) allow us to identify giant planets signals as well as to discriminate the high activity levels of the host stars, enabling an overall monitoring of stellar activity and simultaneous detection of atomic and molecular species in planetary atmospheres.

In this paper, we present the RV measurements of a debated HJ around BD+20 1790 (V429 Gem, K5Ve, $V=9.9$, Jeffries 1995), a very active star $\left(\log R_{H K}^{\prime}=-3.7\right.$, Hernán-Obispo et al. 2015, hereafter HO15) and probable member of the AB Dor moving group (Torres et al. 2008) with an adopted age of $149_{-19}^{+51}$ Myr (Bell et al. 2015). After dedicated spectroscopic observations, Hernán-Obispo et al. (2010; hereafter HO10) interpreted the RV variation of this target as being due to the presence of a massive HJ with a period of 7.8 days. Figueira et al. (2010b) questioned the planet, providing CORALIE RVs showing a clear correlation with the bisector span (BIS), thus attributing the RV variations to photospheric processes. Later, HO15 reported a new RV and activity study exploiting a larger spectroscopic and photometric dataset supporting again the presence of a HJ around BD+20 1790. Finally, Gagné et al. (2016) published a small number of CSHELL NIR RVs with no conclusive results.

This paper is organized as follows: we describe the instruments used in Sect. 2, then we present the observations and the characteristics of our dataset in Sect. 3. In Sect. 4 we show the analysis of both the spectroscopic and photometric time series, suggesting a theoretical model to explain the observed stellar behaviour in the VIS and NIR bands in Sect. 5. Finally we draw our conclusions in Sect. 6.

\section{Instruments}

In this section we summarize the characteristics of the instrumentation used to collect the data of BD+20 1790, with particular emphasis on the new TNG facility GIARPS. In 2012 the high resolution spectrograph HARPS-N (Cosentino et al. 2014) was installed at the Nasmyth-B focal station of the TNG. HARPS-N works in the visible range $(0.39-0.68 \mu \mathrm{m})$ with a resolution of $R=115000$. In the same year, the NIR high resolution spectrograph GIANO (Oliva et al. 2006), working in the wavelength range from 0.97 to $2.45 \mu \mathrm{m}$ at a resolution of R 50000 , was installed and commissioned at the Nasmyth - A focal station of the TNG in 2014. Although designed for direct light feed from the telescope, in its first phase GIANO was fed by fibers. Through the "WOW" (a Way to Others Worlds) Progetto Premiale funding scheme, the Italian National Institute for Astrophysics (INAF) proposed to move GIANO to the Nasmyth$\mathrm{B}$ (re-naming the instrument GIANO-B) and to carry out its simultaneous use with HARPS-N aiming to achieve high resolution spectroscopy in a wide wavelength range $(0.383-2.45 \mu \mathrm{m}$ with a small gap between $\sim 0.7$ and $0.950 \mu \mathrm{m}$ ) obtained in a single exposure. The focus change, made in 2016, also allowed us to restore the original direct feeding from the telescope (Tozzi et al. 2016) and the coupling with HARPS-N. This was the beginning of the observing mode called GIARPS (GIANO-B \& HARPS-N, Claudi et al. 2017). The two spectrographs are still able to work separately, so it is possible to have three different observing modes: a) HARPS-N only; b) GIANO-B only; c) GIARPS, by splitting the light with a dichroic. GIARPS uses both the instruments for high precision RV measurements, exploiting the simultaneous reference technique with HARPS$\mathrm{N}$ in the visible (providing a RV precision $\sigma_{\mathrm{RV}}$ of $\sim 1.0 \mathrm{~m} \mathrm{~s}^{-1}$ ) and a cross-correlation function (CCF) method based on telluric lines with GIANO-B $\left(\sigma_{\mathrm{RV}} \sim 10 \mathrm{~m} \mathrm{~s}^{-1}\right.$, Carleo et al. 2016). The introduction of an absorption gas cell (Seeman et al. 2014), foreseen at the end of 2018, will allow us to reach a better precision $\left(\sim 3 \mathrm{~m} \mathrm{~s}^{-1}\right)$ in the NIR, since the spectral features of a gas cell are more reliable in comparison with the instability of the telluric spectrum. Because of its characteristics, GIARPS can be considered the first and unique worldwide instrument providing not only high resolution but also high precision radial velocity measurements in such a large wavelength range between $B$ and $K$ bands.

The Immersion Grating Infrared Spectrometer (IGRINS; Mace et al. 2016) is the cross-dispersed NIR spectrograph, mounted alternatively at the Harlan J. Smith 2.7 m telescope 
Table 1. Summary of the spectroscopic data presented in this work.

\begin{tabular}{lcccccc}
\hline \hline Instrument & $\mathrm{N}_{\text {spectra }}$ & $\begin{array}{c}\text { Spect. range } \\
(\mu \mathrm{m})\end{array}$ & $\mathrm{S} / \mathrm{N}$ & $\begin{array}{c}\sigma_{R V} \\
\left(\mathrm{~km} \mathrm{~s}^{-1}\right)\end{array}$ & $\begin{array}{c}\mathrm{RV} \mathrm{rms} \\
\left(\mathrm{km} \mathrm{s}^{-1}\right)\end{array}$ & $\begin{array}{c}\text { Peak-to-valley } \\
\left(\mathrm{km} \mathrm{s}^{-1}\right)\end{array}$ \\
\hline GIANO/GIANO-B & 18 & $0.95-2.45$ & 72 & 0.036 & 0.130 & 0.384 \\
HARPS-N & 20 & $0.38-0.69$ & 35 & 0.029 & 0.280 & 1.036 \\
IGRINS & 29 & $1.45-2.5$ & 134 & 0.059 & 0.109 & 0.384 \\
\hline
\end{tabular}

Notes. For each dataset we list the instrument used for the observations, the number of spectra, the spectral range, the typical S/N, the RV nominal internal error $\left(\sigma_{R V}\right)$, the RV rms scatter, and the peak-to-valley value of the RVs.

(McDonald Observatory, TX, USA) and at the $4.3 \mathrm{~m}$ Discovery Channel Telescope (Lowell Observatory, AZ, USA), with a resolving power of $R=45000$ (Yuk et al. 2010; Park et al. 2014). It covers the $H$ and $K$ windows, from 1.45 to $2.5 \mu \mathrm{m}$ in a single acquisition. REM (Rapid Eye Mount; Chincarini et al. 2003) is a $60 \mathrm{~cm}$ robotic telescope located at the La Silla station of the European Southern Observatory (ESO, Chile). The telescope hosts two instruments: REMIR, an infrared imaging camera, and ROS2, a visible imager. The two cameras can also observe simultaneously the same field of view thanks to a dichroic placed in front of the telescope focus. The ROS2 camera is equipped with a back-illuminated CCD $(2048 \times 2048$ pixels, 13.5 micron pixels size, $0.58 \mathrm{arcsec} /$ pixel plate scale), which has a corrected $9.8 \times 9.8$ arcmin field of view, and observes simultaneously through the four Sloan/SDSS $g, r, i, z$ filters. The REMIR camera is equipped with a Hawaii I CCD $(512 \times 512$ pixels, $1.2 \mathrm{arcsec} /$ pixel plate scale $)$, which has a corrected $10 \times 10$ arcmin field of view, and it is equipped with $z J H K$ filters.

\section{Observations and data reduction}

In this section we describe the data acquired from each instrument. The spectroscopic datasets are presented in Sects. 3.1-3.3, and a complete summary of them, including the uncertainties of the RVs measurements (obtained as explained before), the typical signal-to-noise ratio $(\mathrm{S} / \mathrm{N})$, the $\mathrm{RV} \mathrm{rms}$, and the peakto-valley amplitude, is reported in Table 1. The photometric observations are described in Sect. 3.4.

\subsection{GIANO/GIANO-B}

We collected spectra of BD+20 1790 with TNG instrumentation in three different observing campaigns (see Table 1). The first dataset consists of 18 observations with GIANO (March 28-April 3, 2016), supported by quasi-simultaneous photometry with the REM telescope (see below). The second dataset (seven spectra) was acquired during the commissioning of GIANO-B in November 2016. The third one contains five spectra of GIANOB obtained during the GIARPS commissioning in March 2017. Two of them are acquired in GIARPS mode in order to test the simultaneity of GIANO-B and HARPS-N observations. One additional HARPS-N spectrum was later collected without the NIR counterpart because GIANO-B was temporarily unavailable.

GIANO data were reduced with the IRAF package ECHELLE and the dedicated scripts collected in the GIANO_TOOLS ${ }^{1}$ package, while GIANO-B spectra are processed with the dedicated pipeline GOFIO (Rainer et al., in

\footnotetext{
1 Available at the TNG webpage: http://www.tng.iac.es/ instruments/giano/giano_tools_v1.2.0.tar.gz
}

prep.). We obtained a set of 30 NIR RVs over one year (listed in Table A.1), with the method described in Carleo et al. (2016), in which the telluric lines are used as wavelength reference and the CCF method is used to determine the stellar RV. For this purpose, we constructed two suitable digital masks that include about 2000 stellar lines and a similar number of telluric lines. After the correction of the spectra to the barycentre of the solar system, the procedure performs the cross correlation of individual orders of the normalized spectra with the appropriate masks (both stellar and telluric), with the derivation of individual CCF. Through a weighted sum of the CCFs of the individual orders, we obtain the final stellar and telluric CCFs. These are fitted with Gaussian profiles to derive the stellar and telluric RV, respectively. The latter are finally subtracted from the former, providing the relative stellar RVs. The uncertainties are then evaluated taking into account the photon statistics. In the present analysis we consider a slightly different approach, which takes into account the weight of the single orders, some of them being affected by telluric lines and thus contributing in different ways to the determination of the RVs. Starting from the $\mathrm{RV}$ of individual orders, we calculate the weighted average RV for each exposure and its corresponding error. As a final step, we derive the bisector velocity span (BIS, as in Carleo et al. 2016) of the CCF and we calculate the uncertainties on this quantity by considering the fractions of the CCF used for the derivation of the BIS, resulting in $\sqrt{10} \sigma_{\mathrm{RV}}$, where $\sigma_{\mathrm{RV}}$ is the RV error.

\section{2. $H A R P S-N$}

HARPS-N RVs are extracted with the usual data reduction software (DRS, Pepe et al. 2002) by cross-correlating the observed spectrum with a numerical mask that depicts the spectral features of a $\mathrm{K} 5$ star. To work with uniform RV values, we processed our HARPS-N spectra and the ones collected by HO15 (except for one spectrum with very low S/N at JD 2456681) with the current HARPS-N DRS through the YABI workflow (Hunter et al. 2012) installed at IA2 ${ }^{2}$ at the INAF Observatory of Trieste. Since this facility allows us to customize the re-processing, we enlarge the width of the $\mathrm{CCF}$ to take into account the quite large $v \sin i$ of this star $\left(10.03 \pm 0.47 \mathrm{~km} \mathrm{~s}^{-1}\right.$, López-Santiago 2005) and the consequent line broadening. The resulting RVs are listed in Table A.2, together with the CaII activity indicator, $\log R^{\prime}{ }_{H K}$, obtained with the dedicated tool of the HARPS-N DRS (the method is provided in Lovis et al. 2011) also available on YABI, and the BIS (see e.g. Queloz et al. 2001) estimated as in Lanza et al. (2018), starting from the computed CCF of the DRS. The usually adopted uncertainty for the BIS is twice the value of the RV uncertainty, on the basis that the BIS slope is calculated using the top and lower half of a single line measurement. However, this occurs in the ideal case in which the bins of the CCF used for the estimation of the bisector

2 http://ia2.inaf.it 
are not correlated, therefore, in our case the multiplying factor is increased to 2.5 to avoid an underestimation of the uncertainties (see the justification in Lanza et al. 2018).

\subsection{IGRINS}

The 29 spectra of BD+20 1790 collected with IGRINS over one year, from April 2016 to March 2017, are reduced with the IGRINS Pipeline Package ${ }^{3}$. We acquired three different datasets, since the run with IGRINS installed at the Discovery Channel Telescope (DCT) occurred in between the two runs at the McDonald (McD) Observatory (see Table 1). The RV measurements (Table A.3) are obtained with the same procedure used for GIANO.

\subsection{REM}

During the first run with GIANO we obtained quasisimultaneous photometry with REM from May 30 to April 4, 2016. We used tasks within IRAF for bias correction and flat fielding, and the technique of aperture photometry to extract magnitude time series for BD+20 1790 and for other stars detected in the frames, which were selected as candidate comparison stars. In particular we identified two stars that were found to be non-variable and were used as comparison (C) and check (CK) stars (C: 2MASS 07233899+2025102, J $=11.23 \mathrm{mag}, H=11.43 \mathrm{mag}, K=11.17 \mathrm{mag}$; CK: $2 \mathrm{MASS}$ $07234597+2025328, J=12.15 \mathrm{mag}, H=11.97 \mathrm{mag}, K=$ $11.91 \mathrm{mag})$. We measured a standard deviation $\sigma_{C-C K}=$ $0.014 \mathrm{mag}$ in their differential light curve. The visible and infrared magnitudes of BD+20 1790 were computed differentially with respect to the comparison star. After averaging the six consecutive differential magnitudes obtained on each night with ROS2, we obtained a time series of six average griz-band differential magnitudes and four $J H K$-band differential magnitudes for the subsequent analysis. The average standard deviation associated with the nightly averaged magnitudes, which we consider as our photometric precision, is $\sigma<0.015$ mag for all filters, and $\sigma \simeq 0.04$ mag for the $H$ and $K$ magnitudes.

\section{Data analysis}

\subsection{Spectroscopic data}

First, we reproduced the orbital fit of the VIS RVs as presented in HO15 (with SARG, FOCES, and HERMES data only) with a Keplerian function ${ }^{4}$ as in Desidera et al. (2011). Our model is displayed in the upper panel of Fig. 1 as a dashed line together with the VIS data used for the fit, represented by grey dots. The obtained RV semi-amplitude is $926.8 \pm 34.6 \mathrm{~ms}^{-1}$ and the period is $7.7827 \pm 10^{-4}$ days, in good agreement with the result proposed by HO15. As in HO15, we were not able to obtain a solution including their HARPS-N data so we only overimposed them to the fitting function. We observe a match that by itself casts some doubt on a Keplerian interpretation of the RV variation.

Our phase-folded NIR RVs are shown as well in Fig. 1 (lower panel): GIANO and GIANO-B RVs (red dots) show an rms scatter of $129.6 \mathrm{~ms}^{-1}$, while IGRINS RVs (light blue dots) show an rms scatter of $109.2 \mathrm{~ms}^{-1}$. The rms scatter of the whole

\footnotetext{
3 https://github.com/igrins/plp

4 We adopted the IDL least-squares MPFIT package available at http: $/ /$ purl.com/net/mpfit
}

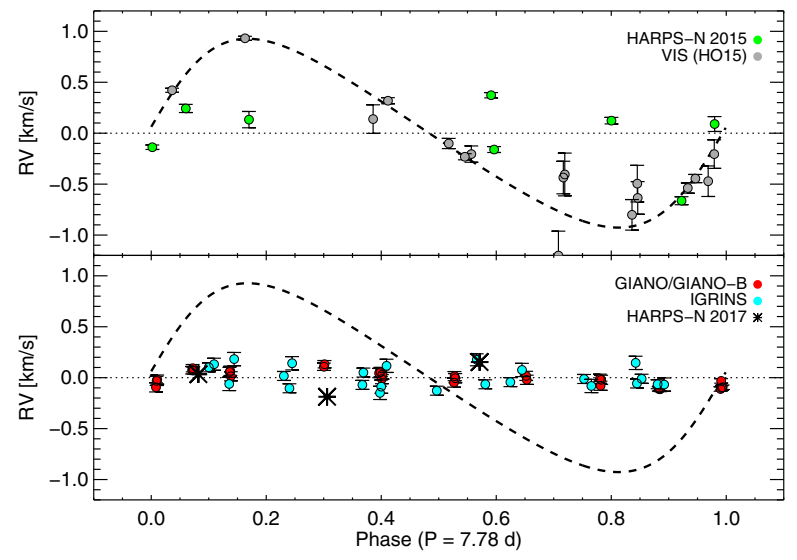

Fig. 1. Orbital fit at 7.78 days found by HO15 compared to phasefolded visible and NIR RVs. Top panel: orbital fit (black dashed line) obtained with the visible data (FOCES, SARG, and HERMES RVs from HO15, grey dots) and HARPS-N 2015 RVs (green dots). Bottom panel: orbital fit (black dashed line), GIANO/GIANO-B (red dots), IGRINS (light blue dots), and HARPS-N 2017 (black asterisks, two acquired in GIARPS mode) RVs.

Table 2. Summary of the values of Spearman $(\rho)$ correlation coefficients and corresponding $p$-values between $\mathrm{RVs}$ and activity indicators.

\begin{tabular}{lll}
\hline \hline Parameters & $\rho$ & $p$-value \\
\hline HARPS-N (2015, 2017): & & \\
RV - BIS & -0.92 & $2 \times 10^{-8}$ \\
RV residuals - BIS & 0.39 & 0.13 \\
RV - log $R_{H K}^{\prime}$ & 0.23 & 0.45 \\
RV - vasy(mod) & 0.89 & $2 \times 10^{-7}$ \\
RV - $\Delta V$ & -0.91 & $1 \times 10^{-7}$ \\
RV - H $\alpha$ & -0.35 & 0.22 \\
\hline GIANO, GIANO-B: & & \\
RV - BIS & -0.14 & 0.44 \\
RV - HeI & -0.08 & 0.65 \\
\hline IGRINS: & & \\
RV - BIS & 0.13 & 0.48 \\
RV - Br $\gamma$ & 0.12 & 0.51 \\
\hline
\end{tabular}

NIR dataset is $119 \mathrm{~ms}^{-1}$. Finally, the black asterisks represent new HARPS-N data (two of them have the NIR simultaneous counterpart). Figure 1 shows that the amplitude of NIR RVs (calculated as the difference between maximum and minimum RVs) is $437.3 \mathrm{~ms}^{-1}$, four times lower with respect to the optical one reported in $\mathrm{HO} 15$ and interpreted as a signature of a hot Jupiter. Therefore, according to our data we can exclude any companion with those characteristics, ascribing the observed variation to phenomena of stellar origin.

Actually, the Spearman rank correlation between RVs and $\mathrm{BIS}$, in this case for the whole HARPS-N dataset (HO15 and the three epochs presented here) is -0.92 , with a very high statistical significance $\left(p\right.$-value $=2 \times 10^{-8}$, evaluated through the IDL Astronomy Library routine SAFE_CORRELATE; see Table 2 for a summary of the measured correlations between RVs and activity indices of the whole dataset), showing an unambiguous linear trend (Fig. 2). After subtracting this correlation from the HARPS-N RVs time series, the resulting residuals show an rms of $61.1 \mathrm{~ms}^{-1}$ and the Generalized Lomb-Scargle (GLS) periodogram (Zechmeister \& Kürster 2009) does not show any 


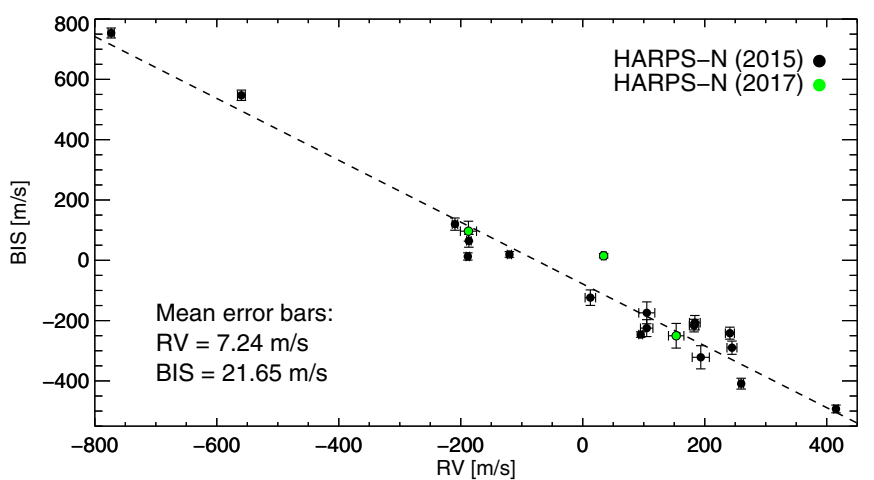

Fig. 2. Correlation between RVs and BIS for HARPS-N data (HO15 and the three new epochs in this work).

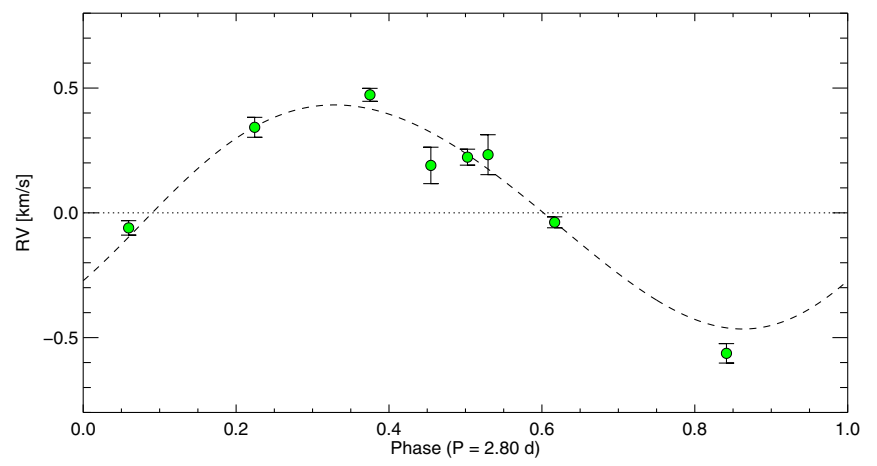

Fig. 3. Phase-folded HARPS-N RVs (2015, reprocessed from HO15 dataset) at stellar rotational period.

significant periodicity (i.e. with an amplitude larger than four times the standard deviation of the power spectrum, corresponding to a false alarm probability (FAP) larger than 0.01). As further evidence that the original HARPS-N RVs are modulated by the stellar rotation of $2.801 \pm 0.001 \mathrm{~d}$ (the photometric period by $\mathrm{HO} 10$ ), in Fig. 3 we fit them with a Keplerian function by using as a first guess that period, obtaining a very good agreement with our model $\left(P=2.80150 \pm 2 \times 10^{-5} \mathrm{~d}, K=\right.$ $\left.0.449 \pm 7.370 \mathrm{~km} \mathrm{~s}^{-1}, e=0.065 \pm 0.018\right)$.

We also investigated the other activity indices available from HARPS-N, for example $\log R_{H K}^{\prime}$ as described in Sect. 3, and the CCF asymmetry indices: the $\Delta \mathrm{V}$ (a measure of the RV shift produced by the exclusive contribution of the asymmetry of the CCF; see Nardetto et al. 2006 and Figueira et al. 2013) and the $V_{\text {asy(mod) }}$ (the modified version of the index $V_{\text {asy }}$ by Figueira et al. 2013 for which the dependence from the RV shift is removed), presented in Lanza et al. (2018). ${ }^{5}$ Finally, we also checked the correlation with the $\mathrm{H} \alpha$ index derived as in Sissa et al. (2016). As for the BIS, the other asymmetry indices show significant correlation with RVs, while we find weak correlations with $\log R_{H K}^{\prime}$ and $\mathrm{H}_{\alpha}$ indicators (Table 2).

We then focused our attention on the NIR data. We computed the GLS for both GIANO and IGRINS RVs even if the sampling is not suitable for a proper periodogram analysis (too few and too sparse points) or for a proper resolution of the known photometric period. The periodograms are quite noisy and do not exhibit any significant periodicity. Only for GIANO did we investigate the FAP (estimated by generating 10000 artificial RV

\footnotetext{
5 See a description of the $V_{\text {asy(mod) }}$ in the poster "Line asymmetry indicators to detect stellar activity effects in radial velocity measurements" by Lanza et al., available at: https://sites.google.com/a/yale. edu/eprv-posters/home.
}

curves obtained from the real one, keeping the epochs of observations fixed but making random permutations of the RV values) of a peak at $7.74 \mathrm{~d}$ responsible for the apparent signal of the GIANO data when phase-folded with the orbital period proposed by HO15 (red dots in Fig. 1, lower panel). This test returned a FAP of $24.4 \%$, so this periodicity is probably produced by random noise. We also noticed a small amount of power corresponding with the photometric period of the star, but it appears to be related to the GIANO data sampling according to the analysis of the window function. No prominent periodicity is found either when examining the GLS of the whole NIR dataset, which is obtained by applying a quite negligible RV offset between the two instruments $\left(5 \mathrm{~m} \mathrm{~s}^{-1}\right)$.

In order to investigate a possible correlation between NIR RVs and activity indicators, we first measured the Spearman coefficient for GIANO and GIANO-B data, in particular between the BIS and the RVs, the BIS and HeI index at $1.083 \mu \mathrm{m}$ (extracted as in Robertson et al. 2016), and between the HeI index and the RVs, but we found no strong correlation (Table 2), mainly due to the uncertainties in the measurements. A similar analysis was performed for the IGRINS data. Since the HeI line is out of the spectral coverage of IGRINS, we investigated the impact of the activity on RVs through the Brackett $\gamma(\mathrm{Br} \gamma)$ emission line at $2.16 \mu \mathrm{m}$ (not available in the GIANO spectra because of the discontinuity among the orders), extracted as in Robertson et al. (2016). As in the case of GIANO, no significant correlation was found between RVs and these indicators (Table 2). Apparently our NIR radial velocities for BD+20 1790 are not highly sensitive to activity.

\subsection{Photometric data}

We used the GLS and the CLEAN (Roberts et al. 1987) periodogram analyses to search for significant periodicities in the $\mathrm{BD}+201790$ photometric time series related to its rotation period. As an example, the GLS and CLEAN periodograms are plotted in Fig. 4 for the case of the $g$ filter. The solid black line represents the normalized power versus period, whereas the dotted red line is the spectral window function. The horizontal red dashed line represents the power level corresponding to a FAP $=0.01$ (confidence level of $99 \%$, obtained with 1000 moke light curves as in Sect. 4.1).

Our periodogram analysis of the photometric variation confirmed the already known stellar rotational period $P=2.76 \pm$ 0.04 days (corresponding to the most powerful and significant peak in the periodogram, indicated with a red mark), slightly lower than the literature value possibly because of the short baseline of the observations (six consecutive nights) or the effect of differential rotation. The uncertainty on the period is calculated following the prescription of Lamm et al. (2004). We note that the secondary power peak in the GLS periodogram is absent in the CLEAN periodogram, which has the capability of effectively removing beat frequencies arising from the data sampling.

In Fig. 5 we plot the differential light curves of BD+20 1790 phased with the rotation period $P=2.76 \mathrm{~d}$, using different colours for different filters. The solid lines represent sinusoidal fits to the phased magnitude computed using the rotation period. The peak-to-peak amplitudes of the sinusoidal fits measured in different filters are reported as labels in the figure. We observe that the REM light curves show different amplitudes in the different bands: unexpectedly, the amplitudes at longer wavelengths are greater than those at shorter wavelengths and the NIR modulation is almost in anti-phase with respect to the optical modulation in the gri passbands (the maximum of the light 

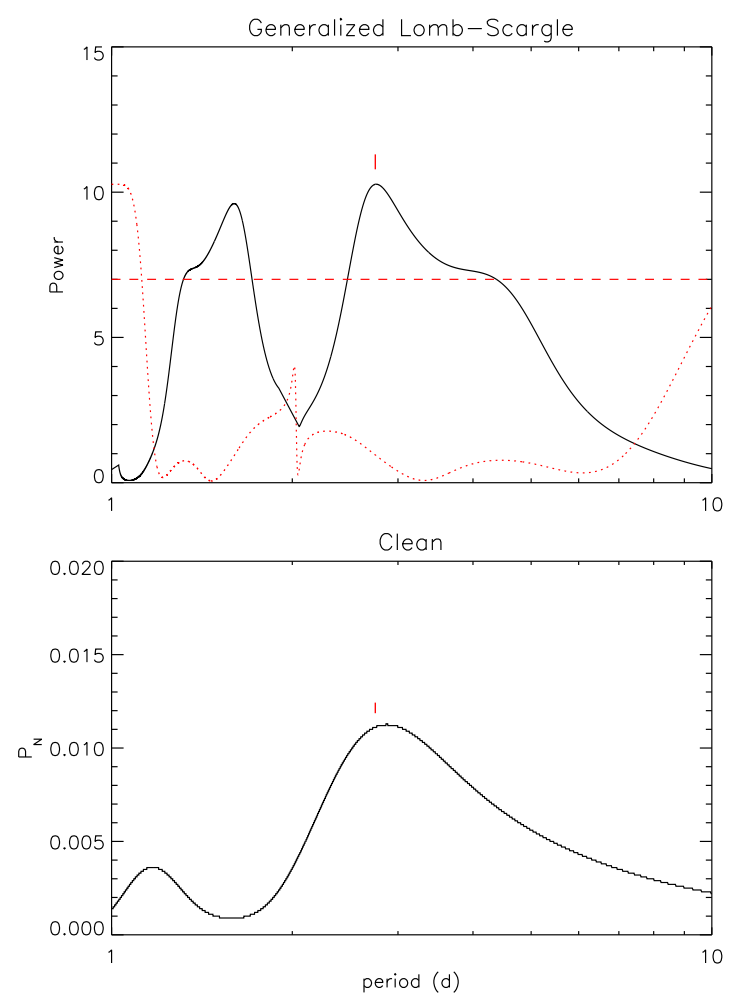

Fig. 4. Top panel: generalized Lomb-Scargle periodogram of the $g$-band photometric time series of BD+20 1790. The solid black line is the normalized power versus period, the dotted red line is the spectral window, and the horizontal dashed line indicates the power level corresponding to a FAP $=0.01$. Bottom panel: CLEAN periodogram. The red mark is the most powerful and significant peak in the periodogram.

curves are located at $\sim 0.8$ for the gri filters, at $\sim 0.1$ for $z$ and at $\sim 0.2$ for JHK). Moreover, the amplitudes of the optical light curves are a factor of approximately seven smaller than in other seasons (cf. the light curves in Fig. 3 in HO15). This could be due to a transient phase of peculiar activity during the REM observing season (that covered a very short period) with respect to what was observed in past campaigns. This particular variability is also supported by the comparison between our HARPS-N RVs and the ones in HO15, as noticed also by Figueira et al. (2010b) with CORALIE data.

\section{Discussion}

The present investigation adds another important piece of evidence to the multi-wavelength characterization of the RV variations in late-type stars. Our results show that the amplitude of the NIR RV modulation in BD+20 1790 is generally a factor of 2-3 smaller than in the optical band, in agreement with the results obtained by Crockett et al. (2012) for very active young stars. This indicates that brightness inhomogeneities, whose contrast is generally smaller in the NIR than in the optical passband, are mainly responsible for the RV variations via line profile distortions. Other effects, such as quenching of convective blueshifts (e.g. Lanza et al. 2011) or line profile distortions produced by the Zeeman effect (Reiners et al. 2013), are probably less important in these very active and rapidly rotating ( $v \sin i \geq 10 \mathrm{~km} \mathrm{~s}^{-1}$ ) stars. Nevertheless, an intriguing result is the small amplitude of the RV variations in the NIR as measured by GIANO contemporaneously to the REM observations that show a remarkable NIR rotational modulation in the $J, H$, and
$K$ passbands. To interpret these results, we first consider a simple model for the wide-band photometric variations, including the effects of both dark and bright spots. Several models for the simultaneous photometric and RV variations of late-type stars have been proposed (e.g. Boisse et al. 2012; Dumusque et al. 2014; Herrero et al. 2016). They include the effects of solar-like faculae whose contrast increases towards the limb. Here, we consider a hot spot in the photosphere that has a constant contrast at different limb positions, similarly to the behaviour generally assumed for a cool spot.

From the stellar $v \sin i$, radius, and rotation period (from HO10), we estimated an inclination of the stellar spin axis to the line of sight of $\sim 50^{\circ}$. With a simulation we then reproduced the sinusoidal shapes, amplitude ratio, and phase difference of the optical and the NIR light curves by assuming a circumpolar active region, always in view, consisting of two co-spatial components (as observed in e.g. V410 Tau, Rice et al. 2011): a cool feature (hereinafter "cool spot") covering a fraction $f_{\mathrm{s}}$ of its total area and a hot feature (hereinafter "hot spot") covering the remaining fraction $1-f_{\mathrm{s}}$.

Therefore, the average brightness of the active region at wavelength $\lambda$ can be written as

$I_{\mathrm{a}}=f_{\mathrm{s}} B\left(T_{\mathrm{c}}, \lambda\right)+\left(1-f_{\mathrm{s}}\right) B\left(T_{\mathrm{h}}, \lambda\right)$,

where, for simplicity, we assume that the brightness of each component is given by a Planck function $B(T, \lambda)$, with $T_{\mathrm{c}}$ being the temperature of the cool spot and $T_{\mathrm{h}}$ that of the hot spot. Those temperatures verify the inequality: $T_{\mathrm{c}}<T_{\text {phot }}<$ $T_{\mathrm{h}}$, where $T_{\text {phot }}$ is the temperature of the unperturbed photosphere. The contrast of the active region is $C_{\mathrm{s}}=1-\left(I_{\mathrm{a}} / I_{\text {phot }}\right)$, where $I_{\text {phot }}$ is the brightness of the unperturbed photosphere. For an active region dominated by the cool spot, $C_{\mathrm{s}}>0$ because $I_{\mathrm{a}}<I_{\text {phot }}$, while for an active region dominated by the hot spot, $C_{\mathrm{s}}<0$.

For BD+20 1790, we assume $T_{\text {phot }}=4410 \mathrm{~K}(\mathrm{HO} 15)$, while for the cool and the hot spots we assume temperatures $T_{\mathrm{c}}=$ $T_{\text {phot }}-1000 \mathrm{~K}$ and $T_{\mathrm{h}}=T_{\text {phot }}+1000 \mathrm{~K}$, respectively. Those temperature differences are typical of young and active stars such as Weak T-Tauri stars (cf. Rice et al. 2011; Koen 2016). The contrast is plotted versus the fraction of the active region covered by the cool spot in Fig. 6 for the optical wavelength $\lambda_{\text {opt }}=636 \mathrm{~nm}$ and the NIR wavelength $\lambda_{\mathrm{NIR}}=1705 \mathrm{~nm}$. Those values correspond approximately to the mean wavelengths of the gri bands and of the $J H K$ bands, respectively.

We see that for a restricted range of $f_{\mathrm{s}}$, that is $0.65<f_{\mathrm{s}}<$ 0.67 , the contrast in the optical is negative and small, while that in the NIR is positive and remarkably larger. This leads to a rotational modulation of the optical flux remarkably smaller than, and in anti-phase with, the rotational modulation in the NIR as illustrated by the synthetic light curves in Fig. 7 computed with the model in Sect. 3.2 of Lanza (2016). Specifically, these light curves were computed for an inclination of the spin axis to the line of sight $i=50^{\circ}$, considering an active region of an area of $20 \%$ of that of the star's disc, centred at a latitude of $60^{\circ}$. The quadratic limb-darkening coefficients at the two wavelengths were taken from Claret et al. (2012), while $C_{\mathrm{s}}\left(\lambda_{\mathrm{opt}}\right)=-0.02$ and $C_{\mathrm{s}}\left(\lambda_{\mathrm{NIR}}\right)=0.15$ corresponding to $f_{\mathrm{s}} \simeq 0.665$ in our simple irradiance model (cf. Fig. 6). For simplicity, we assumed that the spot contrasts did not depend on the position on the disc.

The present model is simply illustrative. The amplitudes of the synthesized light curves are remarkably smaller than those observed in BD+20 1790, which may require a larger filling factor of the active region and/or larger temperature contrasts. For 


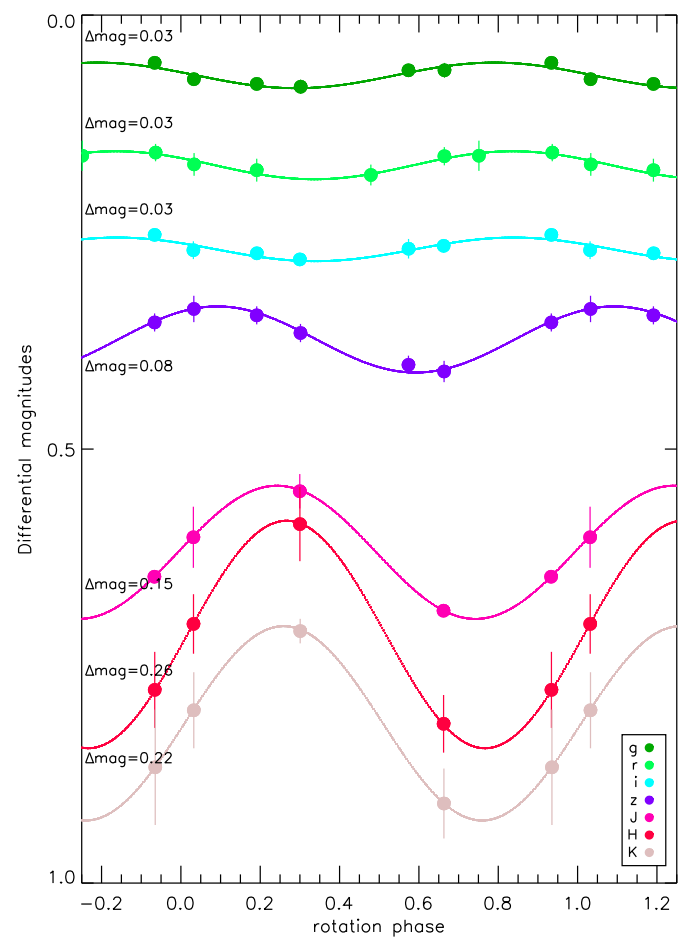

Fig. 5. Differential lightcurves of BD+20 1790 phased with the rotation period $P=2.76 \mathrm{~d}$ in different photometric bands. The solid line is a sinusoidal fit to the data with the same period. Labels show the peak-topeak amplitudes of the lightcurves.

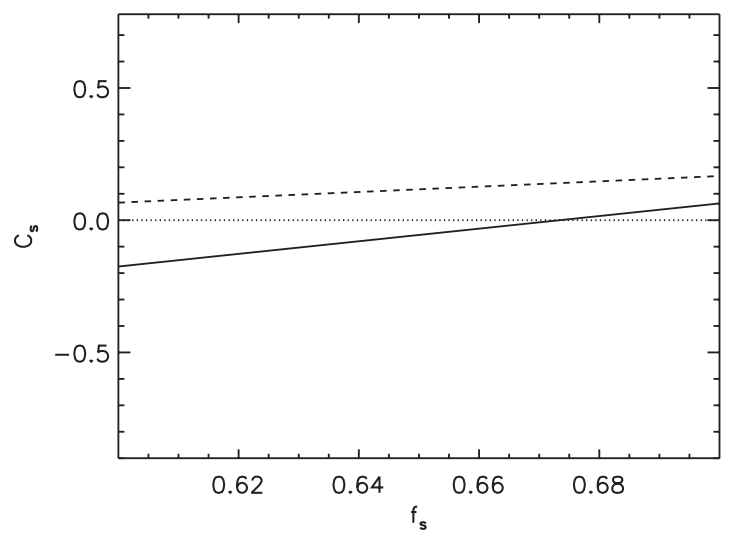

Fig. 6. Contrast of an active region consisting of co-spatial cool and hot spots versus the fraction of its area covered by the cool spot. Two wavelengths are considered: $\lambda_{\mathrm{opt}}=636 \mathrm{~nm}$ (solid line) and $\lambda_{\mathrm{NIR}}=1705 \mathrm{~nm}$ (dashed line)

example, in the case of LkCa 4, Gully-Santiago et al. (2017) found a filling factor as large as $86 \%$, which would imply an amplitude larger by a factor of approximately four in the case of the present model. We finally notice that the variable characteristics of the activity of this star with time might explain why past studies obtained quite different values for the rms scatter of the RVs, and then different interpretations of the nature of this object.

As previously mentioned, the small amplitude of the RV modulation in the NIR as measured by GIANO contemporaneously with REM photometry is intriguing. Assuming that the depth of the spectral lines relative to their adjacent continuum is constant and considering a spot with a contrast $C_{\mathrm{s}}$ at latitude

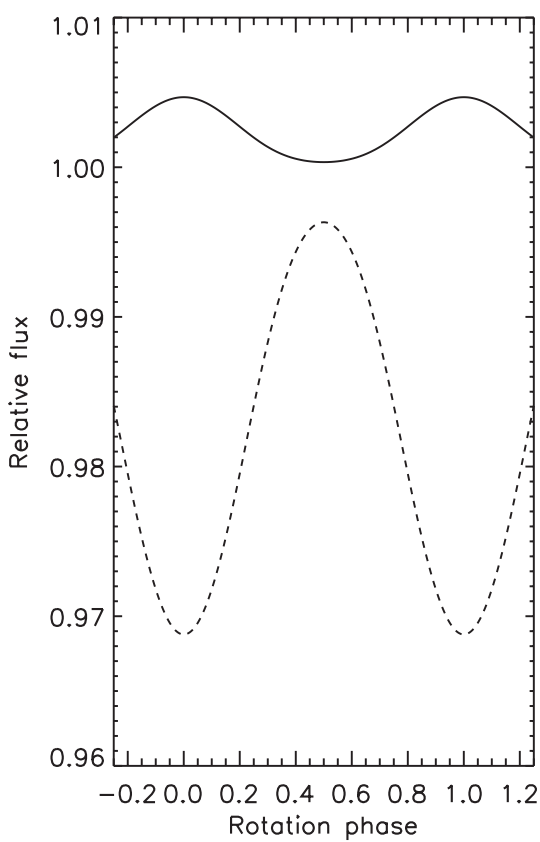

Fig. 7. Light curves showing the rotational modulation of the flux at two wavelengths $\lambda_{\mathrm{opt}}=636 \mathrm{~nm}$ (solid line) and $\lambda_{\mathrm{NIR}}=1705 \mathrm{~nm}$ (dashed line). The amplitudes correspond to a spot having an area of 0.2 of the stellar disc.

$\phi$ with a filling factor $f_{\mathrm{s}}$, we expect an RV modulation approximately of $C_{\mathrm{s}} f_{\mathrm{s}} v \sin i \cos \phi$ (cf. Saar et al. 1997; Desort et al. 2007) that is $\approx 0.75-1.0 \mathrm{~km} \mathrm{~s}^{-1}$ for a spot at $\phi=60^{\circ}$, which is remarkably higher than what has been observed. A cool spot at a higher latitude would reduce the amplitude of the NIR wide-band flux modulations; a quenching of the convective shifts or the Zeeman effect also do not appear to be viable explanations because they increase the effect of a cool spot on the RV at NIR wavelengths (Reiners et al. 2013). Nevertheless, the variation of the relative depths of the spectral lines in the NIR cannot be neglected and it is the dominant effect in the cool spot responsible for the large photometric modulation in the infrared. The relative line depths are strong functions of the continuum opacity and of the degree of element ionization both remarkably varying in a cool spot area with respect to the unperturbed photosphere. In general, these effects produce a remarkable increase of the relative depths of the spectral lines in the cool spot. This compensates for the decrease of the continuum intensity in the spot, reducing the distortions of the spectral line profiles and yielding an RV variation in the NIR smaller than expected from the wide-band photometric variation in the case of constant relative line depths. In any case, a quantitative analysis is not warranted by our data, since a larger number of observations would be required. This scenario suggests a need to better investigate this kind of target, since they might go through specific activity phases during which the VIS and NIR RV amplitudes are similar, possibly resulting in false positives. Looking at the curve phase shifts might give crucial information in these cases (see e.g. the recent result by Hatzes et al. 2018 for the K-giant $\gamma$ Draconis).

\section{Conclusions}

In this paper we present the analysis of RV measurements of the star BD+20 1790, in order to resolve the debate on the presence of a hot Jupiter with an orbital period of 7.8 days, claimed by HO10 and questioned by Figueira et al. (2010b). Since all the 
previous RV measurements were provided by visible spectrographs, we observed this target with the GIANO (in a dedicated run and during the GIANO-B/GIARPS Commissioning) and IGRINS NIR spectrographs to disentangle the origin of the RV variation. The NIR data show a peak-to-valley amplitude of $437.3 \mathrm{~ms}^{-1}$, significantly lower than the VIS one $\left(1853.6 \mathrm{~ms}^{-1}\right)$, demonstrating that the detected RV modulation is due to activity. Therefore, we dismiss the presence of the claimed hot Jupiter around BD+20 1790, which is a very peculiar target from the point of view of stellar activity, as confirmed by our photometric monitoring with REM almost contemporary to the first spectroscopic run with GIANO. Surprisingly, REM light curves show larger amplitudes at longer wavelengths and the NIR curve is almost in anti-phase with respect to the visible one. This has been explained with a photometric model that includes a mixture of cool and hot spots in the same active region.

Multi-band spectroscopy is known to be crucial in the search for exoplanets around young and active stars: here we present the first contribution of a new facility, GIARPS, which adds further value to this method thanks to the simultaneous observations in VIS and NIR bands. Our result clears the current census of hot Jupiters from the only previously known case orbiting a star between 20 to 200 Myr old (Rizzuto et al. 2017; David et al. 2018). The paucity of hot Jupiters in this age range might be explained by the time dependence of planet migration mechanisms (Mann et al. $2018^{6}$ ) or simply by small number statistics. Further investigations are needed to achieve a firm conclusion on this key issue for the evolution of planetary systems.

Acknowledgements. The authors are grateful to the anonymous referee for the careful review and the useful comments that helped to improve the quality of the paper. The authors acknowledge support from INAF through the "WOW Premiale" funding scheme of the Italian Ministry of Education, University, and Research. Based on observations made with the Italian Telescopio Nazionale Galileo (TNG) operated on the island of La Palma by the Fundación Galileo Galilei of the INAF (Istituto Nazionale di Astrofisica) at the Spanish Observatorio del Roque de los Muchachos (ORM) of the IAC. This work used the Immersion Grating Infrared Spectrometer (IGRINS) that was developed under a collaboration between the University of Texas at Austin and the Korea Astronomy and Space Science Institute (KASI) with the financial support of the US National Science Foundation under grant AST-1229522, of the University of Texas at Austin, and of the Korean GMT Project of KASI. These results made use of the Discovery Channel Telescope (DCT) at Lowell Observatory. Lowell is a private, non-profit institution dedicated to astrophysical research and public appreciation of astronomy, and it operates the DCT in partnership with Boston Univ., the Univ. of Maryland, the Univ. of Toledo, Northern Arizona Univ., and Yale Univ. This paper includes data taken with the 2.7-m H. J. Smith Telescope at The McDonald Observatory of The University of Texas at Austin.Based on observations made with the REM Telescope, INAF Chile.

\section{References}

Baruteau, C., Crida, A., Paardekooper, S.-J., et al. 2014, Protostars and Planets VI, 667

Batygin, K., Bodenheimer, P. H., \& Laughlin, G. P. 2016, ApJ, 829, 114 Bell, C. P. M., Mamajek, E. E., \& Naylor, T. 2015, MNRAS, 454, 593

Boisse, I., Bonfils, X., \& Santos, N. C. 2012 A\&A, 545, A109

Bonfils, X., Delfosse, X., Udry, S., et al. 2013, A\&A, 549, A109

Carleo, I., Sanna, N., Gratton, R., et al. 2016, Exp. Astron., 41, 351

Chapman, G. A., \& McGuire, T. E. 1977, ApJ, 217, 657

Chincarini, G., Zerbi, F., Antonelli, A., et al. 2003, The Messenger, 113, 40
Claret, A., Hauschildt, P. H., \& Witte, S. 2012, A\&A, 546, A14

Claudi, R., Benatti, S., Carleo, I., et al. 2017, EPJP, 132, 364

Conod, U., Blind, N., Wildi, F., et al. 2016, Proc. SPIE, 9909, 990941

Cosentino, R., Lovis, C., Pepe, F., et al. 2014, Proc. SPIE, 9147, 91478

Crockett, C. J., Mahmud, N. I., Prato, L., et al. 2012, ApJ, 761, 164

Damasso, M., Bonomo, A. S., Astudillo-Defru, N., et al. 2018, A\&A, in press, DOI:10. 1051/0004-6361/201732459

David, T. J., Mamajek, E. E, Vanderburg, A., et al. 2018, MNRAS 4441157

Desidera, S., Carolo, E., Gratton, R., et al. 2011, A\&A, 533, A90

Desort, M., Lagrange, A.-M., Galland, F., et al. 2007, A\&A, 473, 983

Donati, J. F., Moutou, C., Malo, L., et al. 2016, Nature, 534, 662

Donati, J. F., Yu, L., Moutou, C., et al. 2017, MNRAS, 465, 3343

Dumusque, X., Boisse, I., \& Santos, N. C. 2014, ApJ, 796, 132

Figueira, P., Pepe, F., Santos, N. C., et al. 2010a, EAS Pub. Ser., 42, 125

Figueira, P., Marmier, M., Bonfils, X., et al. 2010b, A\&A, 513, L8

Figueira, P., Santos, N. C., Pepe, F., et al. 2013 A\&A, 557, A93

Gagné, J., Plavchan, P., Gao, P., et al. 2016, ApJ, 822, 402

González-Álvarez, E., Affer, L., Micela, G., et al. 2017, A\&A, 606, A51

Gully-Santiago, M. A., Herczeg, G. J., Czekala, I., et al. 2017, ApJ, 836, 200

Hatzes, A. P., Endl, M., Cochran, W. D., et al. 2018, AJ, 155, 120

Haywood, R. D., Collier Cameron, A., Queloz, D., et al. 2014, MNRAS, 443, 2517

Hernán-Obispo, M., Gálvez-Ortiz, M. C., et al. 2010, A\&A, 512, A45

Hernán-Obispo, M., Tuomi, M., Gálvez-Ortizi et al. 2015, A\&A, 576, A66

Herrero, E., Ribas, I., Jordi, C., et al. 2016, A\&A, 586, A131

Hunter, A., Macgregor, A. B., Szabo, T. O., et al. 2012, Source Code Biol. Med. 7, 1

Jeffries, R. D. 1995, MNRAS, 273, 559

Koen, C. 2016, MNRAS, 463, 4383

Kozai, Y. 1962, ApJ, 67, 592

Lamm, M. H., Bailer-Jones, C. A. L., Mundt, R., et al. 2004, A\&A, 417, 557

Lanza, A. F. 2016, Lect. Notes Phys., (Berlin: Springer Verlag), 914, 43

Lanza, A. F., Boisse, I., Bouchy, F., et al. 2011, A\&A, 533, A44

Lanza A. F., Malavolta L., Benatti S., et al. 2018, A\&A in press, [arXiv: 1804.07039]

López-Santiago, J. 2005, Ph.D. Thesis, Universidad Complutense de Madrid

Lovis, C., Dumusque, X., Santos, N. C., et al. 2011, ArXiv eprints [arXiv: 1107.5325$]$

Mace, G., Kim, H., Jaffe, D. T., et al. 2016, Proc. SPIE, 9908, 99080C

Mahmud, N. I., Crockett, C. J., Johns-Krull, C. M., et al. 2011, ApJ, 736, 123

Najita, J., Carr, J. S., \& Tokunaga, A. T. 1996, ApJ, 456, 292

Nardetto, N., Mourard, D., Kervella, P., et al. 2006, A\&A, 453, 309

Oliva, E., Origlia, L., Baffa, C., et al. 2006, Proc. SPIE, 6269, 626919

Park, C., Jaffe, D. T., Yuk, I., et al. 2014, Proc. SPIE, 9147, 91471

Penn, M. J. 2014, Liv. Rev. Sol. Phys., 11, 2

Pepe, F., Mayor, M., Galland, F., et al. 2002, A\&A, 388, 632

Prato, L., Huerta, M., Johns-Krull, C. M., et al. 2008, ApJ, 687, L103

Queloz, D., Henry, G. W., Sivan, J. P., et al. 2001, A\&A, 379, 279

Quirrenbach, A., Amado, P. J., et al. 2014, Proc. SPIE, 9147, 91471F

Reiners, A., Bean, J. L., Huber, K. F., et al. 2010, ApJ, 710, 432

Reiners, A., Shulyak, D., Anglada-Escudé, G., et al. 2013, A\&A, 552, A103

Reiners, A., Zechmeister, M. Caballero, J. A., et al. 2018, A\&A, 612, A49

Rice, J. B., Strassmeier, K. G., \& Kopf, M., 2011, ApJ, 728, 69

Rizzuto, A. C., Mann, A. W., Vanderburg, A., et al. 2017, AJ, 154, 224

Roberts, D. H., Lehar, J., \& Dreher, J. W. 1987, AJ, 93, 968

Robertson, P., Bender, C., Mahadevan, S., et al. 2016, ApJ, 832, 112

Saar, S. H., \& Donahue, R. A. 1997, ApJ, 485, 319

Sánchez Cuberes, M., Vázquez, M., Bonet, J. A., et al. 2002, ApJ, 570, 886

Scargle, J. D. 1982, ApJ, 263, 835

Seemann U., Anglada - Escudé G., Baade D., et al. 2014, Proc. SPIE Conf. Ser., 9147, 91475

Setiawan, J., Henning, T., Launhardt, R., et al. 2008, Nature, 451, 38

Sissa, E., Gratton, R., Desidera, A. F., et al. 2016, A\&A, 596, A76

Torres, C. A. O., Quast, G. R., Melo, C. H. F., \& Sterik, M.F. 2008, in Handbook of Star Forming Regions, ed. Bo Reipurth, Vol. II: The Southern Sky ASP Monograph Publications, 5, 757

Tozzi, A., Oliva, E., Iuzzolino, M., et al. 2016, Proc. SPIE, 9908, 238

Yu, L., Donati, J.-F., Hébrard, E. M., et al. 2017 MNRAS, 467, 1342

Yuk, I., Jaffe, D. T.; Barnes, S., et al. 2010, Proc. SPIE, 7735, 77351

Zechmeister, M., \& Kürster, M. 2009, A\&A, 496, 577

6 https://keplerscience. arc.nasa.gov/cluster-workshop/ talks/k2clusters-13-andrew-mann.pdf 


\section{Appendix A. Additional tables}

Table A.1. Time series of BD+20 1790 from GIANO and GIANO-B data.

\begin{tabular}{lccccc}
\hline \hline Dataset & JD-2450000 & $\begin{array}{c}\mathrm{RV} \\
\left(\mathrm{km} \mathrm{s}^{-1}\right)\end{array}$ & $\begin{array}{c}\sigma_{R V} \\
\left(\mathrm{~km} \mathrm{~s}^{-1}\right)\end{array}$ & $\begin{array}{c}B I S \\
\left(\mathrm{~km} \mathrm{~s}^{-1}\right)\end{array}$ & $\begin{array}{c}\sigma_{B I S} \\
\left(\mathrm{~km} \mathrm{~s}^{-1}\right)\end{array}$ \\
\hline GIANO & 7475.8302179 & 8.476 & 0.045 & 0.090 & 0.071 \\
& 7475.8387828 & 8.539 & 0.045 & 0.138 & 0.074 \\
& 7475.8473007 & 8.552 & 0.045 & 0.082 & 0.076 \\
& 7476.8292450 & 8.626 & 0.045 & 0.289 & 0.076 \\
& 7476.8378800 & 8.637 & 0.045 & 0.106 & 0.069 \\
& 7476.8463629 & 8.584 & 0.045 & 0.232 & 0.073 \\
& 7478.8578259 & 8.616 & 0.045 & 0.043 & 0.083 \\
& 7478.8768308 & 8.626 & 0.045 & 0.128 & 0.083 \\
& 7478.8946776 & 8.556 & 0.045 & 0.036 & 0.078 \\
& 7478.9032996 & 8.602 & 0.045 & 0.124 & 0.076 \\
& 7479.8734209 & 8.523 & 0.045 & 0.123 & 0.049 \\
& 7479.8819158 & 8.586 & 0.045 & 0.017 & 0.052 \\
& 7479.8917888 & 8.566 & 0.045 & 0.106 & 0.054 \\
& 7480.8530107 & 8.588 & 0.045 & 0.226 & 0.076 \\
& 7480.8612976 & 8.550 & 0.045 & 0.147 & 0.072 \\
& 7481.8545340 & 8.560 & 0.045 & -0.005 & 0.071 \\
& 7481.8631110 & 8.491 & 0.045 & 0.178 & 0.070 \\
& 7481.8715830 & 8.550 & 0.045 & 0.119 & 0.067 \\
\hline GIANO-B 7821.4345852 & 8.474 & 0.036 & -0.007 & 0.085 \\
& 7821.4436589 & 8.498 & 0.036 & -0.014 & 0.084 \\
& 7827.4492982 & 8.440 & 0.036 & 0.332 & 0.087 \\
& 7827.4575966 & 8.460 & 0.036 & 0.274 & 0.090 \\
& 7827.4658833 & 8.437 & 0.036 & 0.094 & 0.092 \\
\hline 7716.7390467 & 8.256 & 0.022 & 0.183 & 0.026 \\
& 7716.7477729 & 8.263 & 0.022 & 0.177 & 0.026 \\
& 7716.7564760 & 8.253 & 0.022 & 0.208 & 0.025 \\
& 7717.5770302 & 8.256 & 0.022 & 0.125 & 0.039 \\
& 7717.5857217 & 8.335 & 0.022 & 0.141 & 0.036 \\
& 7717.5945405 & 8.266 & 0.022 & 0.170 & 0.031 \\
& 7717.6046440 & 8.273 & 0.022 & -0.016 & 0.036 \\
\hline
\end{tabular}

Notes. For each observation we list radial velocities (RV) and the bisector span (BIS) with the corresponding uncertainties 
A\&A 613, A50 (2018)

Table A.2. Time series of BD+20 1790 from HARPS-N data, from HO15 and GIARPS commissioning, uniformly reduced with the new HARPS-N DRS version.

\begin{tabular}{lcccccc}
\hline \hline $\mathrm{JD}-2450000$ & $\begin{array}{c}\mathrm{RV} \\
\left(\mathrm{km} \mathrm{s}^{-1}\right)\end{array}$ & $\begin{array}{c}\sigma_{\mathrm{RV}} \\
\left(\mathrm{km} \mathrm{s}^{-1}\right)\end{array}$ & $\begin{array}{c}B I S \\
\left(\mathrm{~km} \mathrm{~s}^{-1}\right)\end{array}$ & $\begin{array}{c}\sigma_{B I S} \\
\left(\mathrm{~km} \mathrm{~s}^{-1}\right)\end{array}$ & $\log R_{H K}^{\prime}$ & $\sigma_{\log R_{H K}^{\prime}}$ \\
\hline 6293.496128 & 7.818 & 0.004 & 0.019 & 0.009 & -3.982 & 0.002 \\
6293.694395 & 7.750 & 0.004 & 0.065 & 0.021 & -3.984 & 0.003 \\
6293.768482 & 7.726 & 0.005 & 0.120 & 0.020 & -3.995 & 0.003 \\
6346.563090 & 8.021 & 0.003 & -0.247 & 0.009 & -3.944 & 0.002 \\
6347.423110 & 7.374 & 0.007 & 0.547 & 0.017 & -3.977 & 0.004 \\
6347.601234 & 7.163 & 0.005 & 0.754 & 0.017 & -3.989 & 0.004 \\
6348.543400 & 8.113 & 0.008 & -0.217 & 0.021 & -4.016 & 0.007 \\
6348.548909 & 8.113 & 0.010 & -0.207 & 0.024 & -4.041 & 0.008 \\
6348.620652 & 8.167 & 0.009 & -0.290 & 0.022 & -4.018 & 0.008 \\
6348.626160 & 8.166 & 0.008 & -0.242 & 0.020 & -4.022 & 0.007 \\
6349.437201 & 8.017 & 0.015 & -0.174 & 0.036 & -3.946 & 0.011 \\
6349.442721 & 8.027 & 0.011 & -0.225 & 0.028 & -3.955 & 0.008 \\
6679.499824 & 8.346 & 0.004 & -0.493 & 0.013 & -3.972 & 0.003 \\
6679.680352 & 8.177 & 0.004 & -0.409 & 0.018 & -3.887 & 0.002 \\
6682.562820 & 8.137 & 0.015 & -0.322 & 0.038 & -3.941 & 0.011 \\
6682.666891 & 7.945 & 0.009 & -0.124 & 0.026 & -3.966 & 0.007 \\
6796.370888 & 7.748 & 0.003 & 0.013 & 0.012 & -4.030 & 0.003 \\
7821.424255 & 7.690 & 0.013 & 0.097 & 0.033 & -4.023 & 0.009 \\
7823.488210 & 8.031 & 0.013 & -0.250 & 0.041 & -3.946 & 0.009 \\
7827.465395 & 7.915 & 0.004 & 0.015 & 0.008 & -3.991 & 0.003 \\
\hline
\end{tabular}

Notes. For each observation we list radial velocities (RV), $\log R_{H K}^{\prime}$, and the bisector span (BIS) with their related uncertainties. 
Table A.3. Time series of BD+20 1790 from IGRINS data.

\begin{tabular}{|c|c|c|c|c|c|}
\hline Dataset & JD-2450000 & $\begin{array}{c}\mathrm{RV} \\
\left(\mathrm{km} \mathrm{s}^{-1}\right)\end{array}$ & $\begin{array}{c}\sigma_{R V} \\
\left(\mathrm{~km} \mathrm{~s}^{-1}\right)\end{array}$ & $\begin{array}{c}B I S \\
\left(\mathrm{~km} \mathrm{~s}^{-1}\right)\end{array}$ & $\begin{array}{c}\sigma_{B I S} \\
\left(\mathrm{~km} \mathrm{~s}^{-1}\right)\end{array}$ \\
\hline \multirow[t]{7}{*}{ IGRINS@McD } & 7417.80148148 & 8.106 & 0.059 & -0.249 & 0.062 \\
\hline & 7418.77283564 & 8.220 & 0.059 & 0.025 & 0.029 \\
\hline & 7419.81490740 & 8.146 & 0.059 & -0.420 & 0.065 \\
\hline & 7421.84695601 & 8.037 & 0.059 & -0.127 & 0.075 \\
\hline & 7475.63379629 & 8.133 & 0.059 & 0.438 & 0.065 \\
\hline & 7476.61835648 & 8.161 & 0.059 & 0.221 & 0.062 \\
\hline & 7503.59172453 & 8.205 & 0.059 & -0.030 & 0.051 \\
\hline \multirow[t]{10}{*}{ IGRINS@DCT } & 7671.96244213 & 8.285 & 0.065 & 0.362 & 0.054 \\
\hline & 7673.95834490 & 8.015 & 0.065 & 0.270 & 0.056 \\
\hline & 7675.86873842 & 8.178 & 0.065 & 0.339 & 0.047 \\
\hline & 7713.04495370 & 8.219 & 0.065 & 0.143 & 0.060 \\
\hline & 7732.03075231 & 8.248 & 0.065 & 0.117 & 0.054 \\
\hline & 7742.97497685 & 8.244 & 0.065 & 0.464 & 0.078 \\
\hline & 7793.85260416 & 8.021 & 0.065 & 0.228 & 0.043 \\
\hline & 7794.84053240 & 8.035 & 0.065 & 0.345 & 0.033 \\
\hline & 7796.74128472 & 8.041 & 0.065 & 0.181 & 0.072 \\
\hline & 7798.78775463 & 7.953 & 0.065 & 0.396 & 0.047 \\
\hline \multirow[t]{12}{*}{ IGRINS@McD } & 7825.74646990 & 8.018 & 0.044 & 0.318 & 0.063 \\
\hline & 7827.67596064 & 8.131 & 0.044 & -0.125 & 0.053 \\
\hline & 7828.69434027 & 8.046 & 0.044 & 0.136 & 0.056 \\
\hline & 7829.75975694 & 7.958 & 0.044 & -0.053 & 0.071 \\
\hline & 7862.63905092 & 7.964 & 0.044 & -0.101 & 0.053 \\
\hline & 7864.69674768 & 7.973 & 0.044 & 0.144 & 0.055 \\
\hline & 7875.58734953 & 7.925 & 0.044 & 0.026 & 0.053 \\
\hline & 7876.59074074 & 8.079 & 0.044 & 0.213 & 0.055 \\
\hline & 7877.58625000 & 7.902 & 0.044 & 0.134 & 0.055 \\
\hline & 7878.58519676 & 7.986 & 0.044 & 0.179 & 0.058 \\
\hline & 7879.58496527 & 8.017 & 0.044 & -0.006 & 0.053 \\
\hline & 7880.58305555 & 7.965 & 0.044 & -0.023 & 0.052 \\
\hline
\end{tabular}

Notes. For each observation we list radial velocities (RV) and the bisector span (BIS) with the corresponding uncertainties. 
Table A.4. Time series of BD+20 1790 from REM data with different filters.

\begin{tabular}{|c|c|c|c|}
\hline Filter & JD-2450000 & $\begin{array}{c}\Delta m \\
(\mathrm{mag})\end{array}$ & $\begin{array}{c}\sigma_{\Delta m} \\
(\mathrm{mag})\end{array}$ \\
\hline$g$ & $\begin{array}{c}7477.52744 \\
7478.58315 \\
7479.57717 \\
7480.59303 \\
7481.59388 \\
7482.61009\end{array}$ & $\begin{array}{l}-1.960 \\
-1.985 \\
-2.002 \\
-1.984 \\
-2.012 \\
-2.010 \\
\end{array}$ & $\begin{array}{l}0.005 \\
0.004 \\
0.007 \\
0.008 \\
0.009 \\
0.008 \\
\end{array}$ \\
\hline$r$ & $\begin{array}{c}7477.52744 \\
7478.58315 \\
7479.58383 \\
7480.59303 \\
7481.59283 \\
7482.61159 \\
\end{array}$ & $\begin{array}{l}-2.687 \\
-2.722 \\
-2.732 \\
-2.725 \\
-2.753 \\
-2.756 \\
\end{array}$ & $\begin{array}{l}0.013 \\
0.017 \\
0.016 \\
0.012 \\
0.011 \\
0.013 \\
\end{array}$ \\
\hline$i$ & $\begin{array}{c}7477.52822 \\
7478.58392 \\
7479.57717 \\
7480.58715 \\
7481.58804 \\
7482.60679 \\
\end{array}$ & $\begin{array}{l}-3.100 \\
-3.109 \\
-3.129 \\
-3.104 \\
-3.124 \\
-3.122 \\
\end{array}$ & $\begin{array}{l}0.007 \\
0.011 \\
0.007 \\
0.006 \\
0.008 \\
0.010 \\
\end{array}$ \\
\hline$z$ & $\begin{array}{l}7477.52744 \\
7478.58315 \\
7479.57717 \\
7480.59196 \\
7481.59097 \\
7482.61009\end{array}$ & $\begin{array}{l}-3.318 \\
-3.279 \\
-3.344 \\
-3.348 \\
-3.320 \\
-3.409 \\
\end{array}$ & $\begin{array}{l}0.010 \\
0.011 \\
0.010 \\
0.011 \\
0.012 \\
0.015\end{array}$ \\
\hline$J$ & $\begin{array}{l}7479.57544 \\
7480.58707 \\
7481.58691 \\
7482.60688\end{array}$ & $\begin{array}{l}-3.756 \\
-3.839 \\
-3.687 \\
-3.756\end{array}$ & $\begin{array}{l}0.006 \\
0.020 \\
0.005 \\
0.035\end{array}$ \\
\hline$H$ & $\begin{array}{l}7479.57789 \\
7480.58792 \\
7481.58772 \\
7482.60755\end{array}$ & $\begin{array}{l}-3.527 \\
-3.762 \\
-3.575 \\
-3.734\end{array}$ & $\begin{array}{l}0.044 \\
0.043 \\
0.033 \\
0.034\end{array}$ \\
\hline$K$ & $\begin{array}{c}7479.58154 \\
7480.59008 \\
7481.58988 \\
7482.60970\end{array}$ & $\begin{array}{l}-3.850 \\
-4.003 \\
-3.801 \\
-3.904\end{array}$ & $\begin{array}{l}0.067 \\
0.014 \\
0.040 \\
0.044\end{array}$ \\
\hline
\end{tabular}

Notes. For each observation we list the differential magnitudes with the corresponding uncertainty. 